\title{
ON DOMINATING EVEN SUBGRAPHS IN CUBIC GRAPHS*
}

\author{
ROMAN ČADA ${ }^{\dagger}$, SHUYA CHIBA ${ }^{\ddagger}, \mathrm{KENTA} \mathrm{OZEKI}^{\S}$, AND KIYOSHI YOSHIMOTO
}

\begin{abstract}
It is known that a 3-edge-connected graph has a spanning even subgraph in which every component contains at least five vertices, and the lower bound is best possible. A natural question arises of whether we can improve the lower bound by changing the spanning property with the dominating property. In this paper, we show that a 3-edge-connected cubic graph has a dominating even subgraph in which every component contains at least six vertices.
\end{abstract}

Key words. even subgraph, dominating subgraph, 2-factor, cubic graph

AMS subject classifications. 05C38, 05C69

DOI. $10.1137 / 16 \mathrm{M} 1066622$

1. Introduction. In this paper, we consider finite graphs without loops. An even graph is a graph in which every vertex has a positive even degree and a subgraph $H$ of a graph $G$ is said to be dominating if $G-V(H)$ is edgeless. In this paper, a cycle is a connected 2-regular graph and a cycle with $l$ vertices is called an $l$-cycle. A 2 -factor is a spanning 2-regular subgraph of a graph. An edge-cut is a minimal set of edges whose removal increases the number of components of the graph. We call an edge-cut with $l$ edges an $l$-cut. An edge-cut is said to be essential if both of the two new components after deleting it have at least one edge.

For a vertex subset $X \subset V(G)$, the set of edges joining $X$ and $V(G)-X$ is denoted by $\partial(X)$ or simply $\partial X$. If $X$ consists of one vertex $u$, then we denote it simply by $\partial(u)$. For a subgraph $H$ of $G$, we use $\partial H$ instead of $\partial(V(H))$. For terminology and notation not defined in this paper, we refer the readers to [5].

In this paper we consider cubic graphs, i.e., 3-regular graphs. A classical result by Petersen [17] says that a bridgeless cubic graph has a 2 -factor. This well-known result was generalized by Fleischner [10] as follows: a bridgeless graph with minimum degree at least three has a spanning even subgraph in which every component has at least three vertices. If we restrict ourselves to simple graphs, then the lower bound on the order of components is improved to four in [13]. Jackson and Yoshimoto considered 3-edge-connected graphs and showed the following.

Theorem A (Jackson and Yoshimoto [14]). A 3-edge-connected graph with $n$ vertices has a spanning even subgraph in which each component contains at least $\min \{5, n\}$ vertices.

* Received by the editors March 18, 2016; accepted for publication (in revised form) January 9, 2017; published electronically May 18, 2017.

http://www.siam.org/journals/sidma/31-2/M106662.html

Funding: The first author's research was partly supported by the project P202/12/G061 of the Czech Science Foundation and by the project LO1506 of the Czech Ministry of Education, Youth and Sports. The second author's work was supported by JSPS KAKENHI grants 26400187 and 26800083. The third author's work was in part supported by JSPS KAKENHI grant 25871053, by Grant for Basic Science Research Projects from the Sumitomo Foundation and by JST ERATO grant JPMJER1305, Japan. The fourth author's work was supported by JSPS KAKENHI grant 26400190.

${ }^{\dagger}$ Department of Mathematics, University of West Bohemia, Univerzitni 8, 30614 Pilsen, Czech Republic, Centre of Excellence IT, Institute for Theoretical Computer Science, Charles University, 11636 Prague 1, Czech Republic, and European Centre of Excellence NTIS, New Technologies for the Information Society, Technicka 8, 30100 Pilsen, Czech Republic (cadar@kma.zcu.cz).

${ }^{\ddagger}$ Kumamoto University, Kumamoto 860-8555, Japan (schiba@kumamoto-u.ac.jp).

§Yokohama National University, Yokohama 240-8501, Japan (ozeki-kenta-xr@ynu.ac.jp).

『Nihon University, Tokyo 101-8308, Japan (yoshimoto@math.cst.nihon-u.ac.jp). 
They also gave an infinite family of 3-edge-connected cubic graphs in which every 2 -factor contains 5 -cycles. Thus the lower bound in the theorem is best possible in some sense. Kaiser and Škrekovski gave an interesting result, which also generalizes the Petersen's theorem.

Theorem B (Kaiser and Škrekovski [15]). Every graph has an even subgraph which intersects all 3-cuts and 4-cuts.

If a given graph is bridgeless and cubic, then for any $u \in V(G), \partial(u)$ is always a 3 -cut of the graph, and so the above theorem implies the following.

Corollary C. A bridgeless cubic graph has a 2 -factor which intersects all 3 -cuts and 4-cuts.

If a given cubic graph is 3 -edge-connected, then for an $l$-cycle $C$ where $l \in\{3,4\}$, $\partial C$ is an $l$-cut of the graph, and so Corollary $\mathrm{C}$ implies that a 3 -edge-connected cubic graph has a 2-factor in which every component contains at least five vertices.

Matthews and Sumner [16] conjectured that 4-connected claw-free graphs are Hamiltonian and Ryjáček [18] showed the Matthews-Sumner conjecture is equivalent to the conjecture by Thomassen and others $[2,4,19]$ that 4-connected line graphs are Hamiltonian. Fleischner and Jackson [11] showed that the conjecture on line graphs, and so the Matthews-Sumner conjecture, is equivalent to the conjecture by Ash and Jackson [3] that an essentially 4-edge-connected cubic graph has a dominating cycle. Thus it is interesting and important to study the behavior of dominating subgraphs in cubic graphs. In this paper, we prove the following using Corollary C.

TheOREM 1.1. A 3-edge-connected cubic graph has a Hamilton cycle or a dominating even subgraph $F$ such that every component in $F$ contains at least six vertices and $F$ intersects all essential 3-cuts.

In section 2, we give several preparations for the proof of Theorem 1.1, and in section 3 , the proof will be given. Furthermore, we will give remarks on even subgraphs of 3-edge-connected cubic graphs and the traveling salesman problem in section 4 .

We conjecture that Theorem 1.1 can be generalized as in Theorem A.

Conjecture 1.2. A 3-edge-connected graph with $n$ vertices has a dominating even subgraph in which each component has at least $\min \{6, n\}$ vertices.

Also it is a natural question to ask about the lower bound "6" in Theorem 1.1 and Conjecture 1.2.

Problem 1.3. What is the maximum integer $k$ such that any 3-edge-connected graph has a dominating even subgraph in which each component has at least $\min \{k, n\}$ vertices?

The following example implies that the upper bound must be at most nine.

FACT 1.4. There is an infinite family of 3-edge-connected cubic graphs in which every dominating even subgraph has a cycle of order at most nine.

Proof. We construct such a cubic graph. Let $S$ be the graph as in Figure 1, where $S$ has 34 vertices, 49 edges, and 4 "half-edges" whose one end is in $S$. Later we define the other ends of the half-edges.

Let $m$ and $l$ be positive integers with $3 m=4 l$. Let $B$ be $l$ copies of $S$ and $A$ be $m$ mutually disjoint triangles with three half-edges incident to each vertex of the triangle. See Figure 2. Since $A$ and $B$ have $3 m$ and $4 l$ half-edges, respectively, and $3 m=4 l$, we can pair up half-edges in $A$ with half-edges in $B$. It is easy to pair them so that the obtained graph $G$ is 3-edge-connected. 


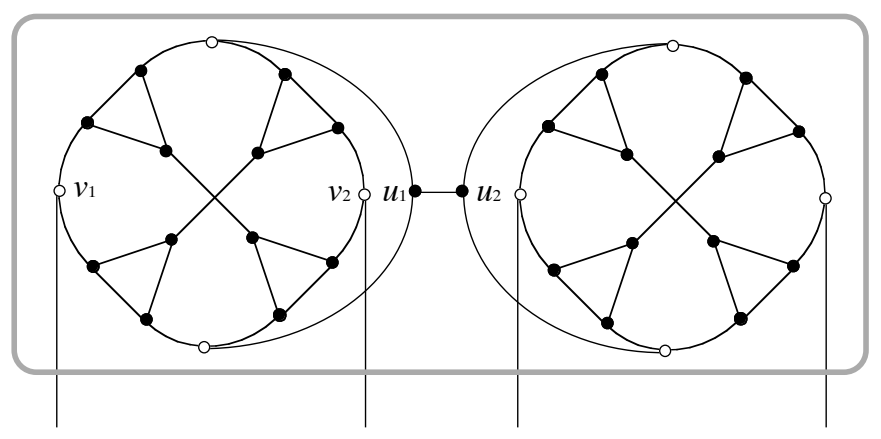

FIG. 1.
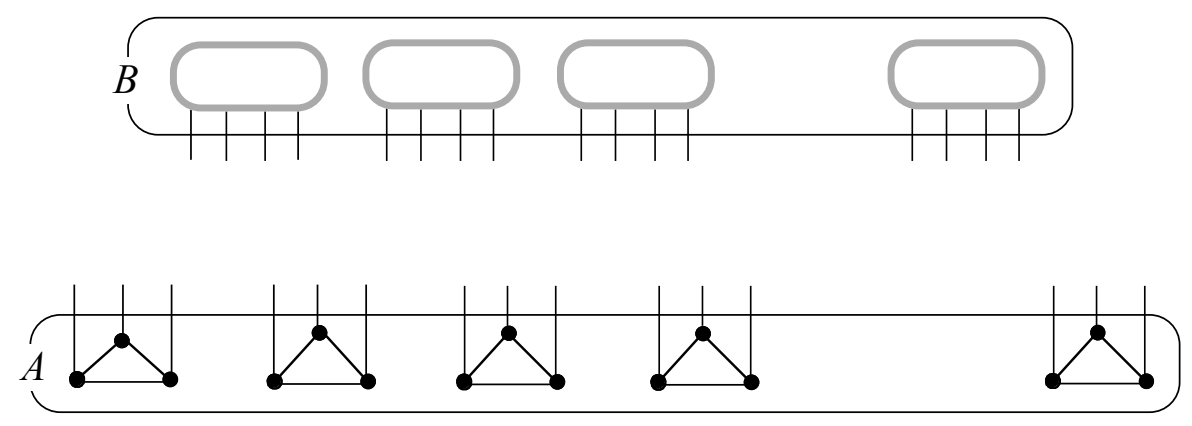

FIG. 2.

We show that any dominating even subgraph $F$ in $G$ has a cycle of order at most nine. If there is a triangle $T$ in $A$ such that $\partial T \cap F=\emptyset$, then obviously $F$ contains the 3-cycle $T$ as a component. Suppose $\partial T \cap F \neq \emptyset$ for all triangles $T$ in $A$. Since $F$ is an even subgraph, $|\partial T \cap F|=2$ for all triangles $T$ in $A$, and so $F$ contains $2 m$ edges joining $A$ and $B$. Since $2 m=8 l / 3$, there is a component $S$ in $B$ such that $|\partial S \cap F|=4$.

Let $u_{1} u_{2}$ be the edge in the middle of $S$; see Figure 1 . Since $F$ is a dominating subgraph in $G$, at least one of the vertices $u_{1}$ and $u_{2}$ is contained in $F$, say, $u_{1}$. Let $L_{S}$ be the left component of $S-u_{1} u_{2}$. Since $F$ is an even subgraph, we have $\left|\partial L_{S} \cap F\right|=2$, and hence $u_{1} u_{2} \notin F$ and $\partial\left(u_{1}\right)-\left\{u_{1} u_{2}\right\} \subset F$. Note that both $v_{1}$ and $v_{2}$ are contained in $F$. Then by the structure of $L_{S}$, it is an easy observation that $v_{1}$ and $v_{2}$ belong to the same component of $F$ which is different from the one containing $u_{1}$, and furthermore the component containing $u_{1}$ has at most nine vertices. This completes the proof of Fact 1.4.

The following question is also natural.

Problem 1.5. Does a 3-edge-connected cubic graph have a dominating even subgraph $F$ such that every component in $F$ contains at least six vertices and $F$ intersects all essential 3 -cuts and 4 -cuts?

2. Preparations. First, we give some additional notation. The set of all the neighbors of a vertex $x \in V(G)$ is denoted by $N_{G}(x)$ or simply $N(x)$ and its cardinality by $d_{G}(x)$ or $d(x)$. For a subgraph $H$ of $G$, we denote $N_{G}(x) \cap V(H)$ by $N_{H}(x)$ and its cardinality by $d_{H}(x)$. For simplicity, we denote $|V(H)|$ by $|H|$ and " $u_{i} \in V(H)$ " by " $u_{i} \in H$." Similarly $G-V(H)$ is denoted by $G-H$. 
(a)

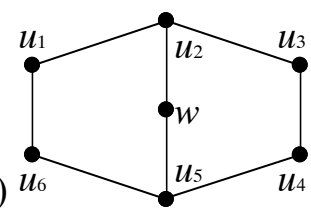

(b)

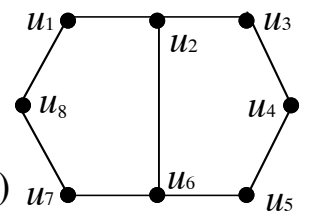

(c)

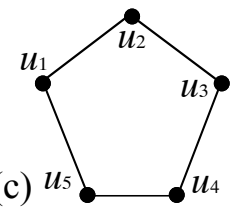

FIG. 3 .

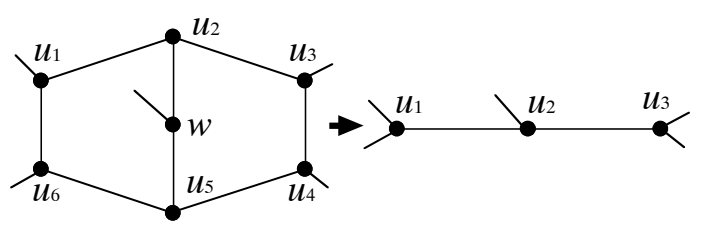

FiG. 4. Reduction of 2-cell.

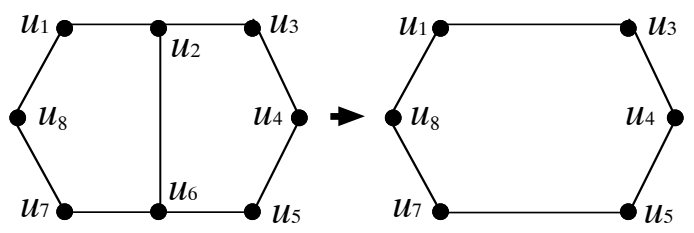

FiG. 5. Reduction of 1-cell.

Recall that an edge-cut is said to be essential if both of the two new components after deleting it have at least one edge. This definition directly implies the following fact, which will be implicitly used in our proofs.

FACT 2.1. For a 2-edge-connected cubic graph $G$ and for a $k$-cut $T$, both of the following hold:

- If $k=2$, then $T$ is always an essential cut.

- If $k=3$ and $T=\partial S$ for some $S \subseteq V(G)$ with $|S| \geq 2$ and $|V(G)-S| \geq 2$, then $T$ is an essential cut.

An $i$-cell is the union of two 5 -cycles in a cubic graph which have $i$ common edges. See Figure 3(a), (b). We call a 5-cycle a 0-cell. In the proof of Theorem 1.1, we will construct a dominating even subgraph from a 2-factor of a cubic graph which is obtained by reducing those cells. Hence we define reductions for those cells first.

Let $D$ be a 2 -cell in $G$, and let $u_{1} u_{2} \cdots u_{6} u_{1}$ be the 6 -cycle and $w$ the remaining vertex in $D$. See Figure 4 . Let $G^{\prime}$ be the graph obtained from $G$ by contracting all of the paths $u_{1} u_{6}, u_{2} w u_{5}, u_{3} u_{4}$ and removing the edges $u_{6} u_{5}$ and $u_{5} u_{4}$. We denote this reduction by $G^{\prime}=G \mid D$.

Let $D$ be a 1 -cell in $G$ and $u_{1} u_{2} \cdots u_{8} u_{1}$ the 8-cycle of $D$. See Figure 5. Let $G^{\prime}$ be the graph obtained from $G$ by removing the edge $u_{2} u_{6}$ and contracting both of the edges $u_{1} u_{2}$ and $u_{7} u_{6}$. We denote by $G^{\prime}=G \mid D$ this reduction. 


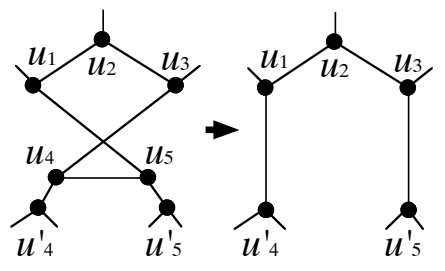

FIG. 6. Reduction of 5-cycle.

Let $D=u_{1} \cdots u_{5} u_{1}$ be a 5 -cycle without chord. Let $u_{i}^{\prime} \in V(G-C)$ which is adjacent to $u_{i}$ for $1 \leq i \leq 5$. See Figure 6 . Let $G^{\prime}$ be the graph obtained from $G$ by removing the edges $u_{1} u_{5}, u_{5} u_{4}, u_{4} u_{3}$ and identifying $u_{1}, u_{4}$ and $u_{3}, u_{5}$, respectively. We denote by $G^{\prime}=\left.G\right|_{u_{2}} D$ this reduction.

We say that a 5-cycle $C$ is good in $G$ if there is an essential 3-cut $T$ in $G$ such that $|T \cap \partial C| \geq 2$. If $C$ has exactly one chord and $|G| \geq 8$, then $C$ is always good because $\partial C$ is an essential 3-cut. If a 2- or 1-cell contains a good 5-cycle, then the cell is also called good. A cell which is not good is called bad. Notice that in a bad cell, every 5-cycle is bad.

We need the following fact in the proof of Theorem 1.1.

FACT 2.2. Let $i \in\{2,1,0\}$. If a 3 -edge-connected cubic graph $G$ has a bad $i$-cell $D$, then $G \mid D$ or $\left.G\right|_{u_{2}} D$ is 3-edge-connected.

This fact is obtained from the following two lemmas.

Lemma 2.3. Let $D=u_{1} u_{2} u_{3} u_{4} u_{5} u_{1}$ be a 5-cycle of a 3-edge-connected cubic graph. If there is an essential 3 -cut $T$ such that $T \cap E(D) \neq \emptyset$, then $D$ is good.

Proof. Suppose $D$ is bad and there is an essential 3-cut $T$ such that $T \cap E(D) \neq \emptyset$. Since $D$ is bad and $G$ is 3-edge-connected, $D$ has no chord. Let $u_{i}^{\prime} \in N_{G-C}\left(u_{i}\right)$ for $1 \leq i \leq 5$. Since $T$ is an essential edge-cut of a cubic graph and $G$ is 3-edge-connected, no pair of edges in $T$ is adjacent, and so $T \cap E(D)$ contains two independent edges, say, $u_{1} u_{2}, u_{4} u_{5}$. Then $\left(T-\left\{u_{1} u_{2}, u_{4} u_{5}\right\}\right) \cup\left\{u_{1} u_{1}^{\prime}, u_{5} u_{5}^{\prime}\right\}$ is an essential 3-cut containing two edges in $\partial D$, a contradiction.

Lemma 2.4. For $k \in\{2,3\}$ and a $k$-edge-connected cubic graph $G$, the following hold:

1. Let $D$ be a 2 -cell and $u_{1} u_{2} \cdots u_{6} u_{1}$ be the 6 -cycle in D. See Figure 4 . If $G \mid D$ is not $k$-edge-connected, then $G$ has an essential $k$-cut containing $\left\{u_{1} u_{2}, u_{5} u_{6}\right\}$ or $\left\{u_{2} u_{3}, u_{4} u_{5}\right\}$.

2. Let $D$ be a 1-cell and $u_{1} u_{2} \cdots u_{8} u_{1}$ be the 8-cycle in D. See Figure 5 . If $G \mid D$ is not $k$-edge-connected, then $G$ has an essential k-cut containing $\left\{u_{1} u_{8}, u_{4} u_{5}, u_{2} u_{6}\right\}$ or $\left\{u_{3} u_{4}, u_{7} u_{8}, u_{2} u_{6}\right\}$.

3. Let $D=u_{1} u_{2} \cdots u_{5} u_{1}$ be a 5-cycle and $u_{j}^{\prime}$ be the vertex in $G-D$ which is adjacent to $u_{j}$ for $1 \leq j \leq 5$. See Figure 6 . If $\left.G\right|_{u_{2}} D$ is not $k$-edge-connected, then $G$ has an essential $k$-cut containing $\left\{u_{1} u_{1}^{\prime}, u_{4} u_{4}^{\prime}\right\}$ or $\left\{u_{3} u_{3}^{\prime}, u_{5} u_{5}^{\prime}\right\}$.

Proof. Let $G^{\prime}=G \mid D$ or $G^{\prime}=\left.G\right|_{u_{2}} D$, respectively, $T$ be a minimum edge-cut of $G^{\prime}$, and $D^{\prime}$ be the subgraph in $G^{\prime}$ corresponding to $D$. Let $S \subset V\left(G^{\prime}\right)$ such that $\partial S=T$ and $u_{1} \in S$. Suppose $|T| \leq k-1$. Since $G$ is $k$-edge-connected, $T$ is not an edge-cut of $G$, and so $T=\partial S$ divides $D^{\prime}$. For a vertex $u \in V(D)$, we denote a vertex in $G-D$ adjacent to $u$ by $u^{\prime}$ if it exists. 
1. Since $\left|T \cap D^{\prime}\right|=1$, by symmetry, we may suppose $T \cap D^{\prime}=\left\{u_{1} u_{2}\right\}$. Since $T$ is a minimum cut, no pair of edges in $T$ is adjacent, and so $\left\{u_{1}^{\prime}, u_{6}^{\prime}\right\} \subset S$. Thus $\partial\left(S \cup\left\{u_{6}\right\}\right)$ is an essential $k$-cut containing $\left\{u_{1} u_{2}, u_{5} u_{6}\right\}$ of $G$.

2. Since $T$ divides $V\left(D^{\prime}\right),\left|T \cap D^{\prime}\right|=2$. By symmetry, we have four cases.

If $T \cap D^{\prime}=\left\{u_{1} u_{3}, u_{5} u_{7}\right\}$, then $\partial S$ is also a $(k-1)$-cut of $G$, a contradiction. If $T \cap D^{\prime}=\left\{u_{1} u_{3}, u_{4} u_{5}\right\}$, then $\partial\left(S \cup\left\{u_{2}, u_{6}\right\}\right)$ is a $(k-1)$-cut of $G$, a contradiction.

If $T \cap D^{\prime}=\left\{u_{1} u_{8}, u_{3} u_{4}\right\}$, then $\partial\left(S-\left\{u_{1}, u_{3}\right\}\right)$ is a $(k-1)$-cut of $G$, a contradiction.

If $T \cap D^{\prime}=\left\{u_{1} u_{8}, u_{4} u_{5}\right\}$, then since $\left\{u_{1}, u_{3}\right\} \subset S, \partial\left(S \cup\left\{u_{2}\right\}\right)$ is an essential $k$-cut containing $\left\{u_{1} u_{8}, u_{4} u_{5}, u_{2} u_{6}\right\}$ of $G$.

3. Since $T=\partial S$ divides $D^{\prime},\left|T \cap D^{\prime}\right|=1$. By symmetry, we may suppose $T \cap D^{\prime}=\left\{u_{1} u_{2}\right\}$. Since $\left\{u_{1}^{\prime}, u_{4}^{\prime}\right\} \subset S$ and $G$ is $k$-edge-connected, $\partial\left(S-u_{1}\right)$ is an essential $k$-cut containing $\left\{u_{1} u_{1}^{\prime}, u_{4} u_{4}^{\prime}\right\}$ of $G$.

Proof of Fact 2.2. If $D$ is a 5-cycle and $\left.G\right|_{u_{2}} D$ is not 3-edge-connected, then by Lemma 2.4, $D$ is good. If $D$ is a 2- or 1-cell and $G \mid D$ is not 3-edge-connected, then there exist a 5-cycle $C$ in $D$ and an essential 3-cut $T$ of $G$ such that $|D \cap T| \geq 2$ by Lemma 2.4. Thus by Lemma 2.3, $D$ is good.

3. Proof of Theorem 1.1. Let $G$ be a 3-edge-connected cubic graph. We may assume that $G$ is not Hamiltonian; otherwise we are done. First we define a sequence of bad cells in $G$ which will be reduced.

Let

$$
\mathcal{D}_{1}=\left\{D_{1}, D_{2}, \ldots, D_{p}\right\}
$$

be a maximal set of mutually disjoint 2-cells in $G$ such that $D_{i+1}$ is bad in $G_{i}$ for each $0 \leq i \leq p-1$, where $G_{0}=G$ and $G_{i}=G_{i-1} \mid D_{i}$ for $1 \leq i \leq p$. If there is no bad 2-cell in $G$, then we define $\mathcal{D}_{1}=\emptyset$ and $p=0$. We denote the subgraph in $G_{i}$ corresponding to $D_{i}$ by $D_{i}^{\prime}$. See Figure 7 . Notice that $G-\bigcup_{l \leq i} D_{l}=G_{i}-\bigcup_{l \leq i} D_{l}^{\prime}$ and, by Fact 2.2, each $G_{i}$ is 3-edge-connected for every $0 \leq i \leq p$. By the maximality of $\mathcal{D}_{1}$, obviously the following claim holds.

ClAim 3.1. There is no 2-cell in $G-\bigcup_{l \leq p} D_{l}=G_{p}-\bigcup_{l \leq p} D_{l}^{\prime}$ which is bad in $G_{p}$.

Let

$$
\mathcal{D}_{2}=\left\{D_{p+1}, D_{p+2}, \ldots, D_{p+q}\right\}
$$

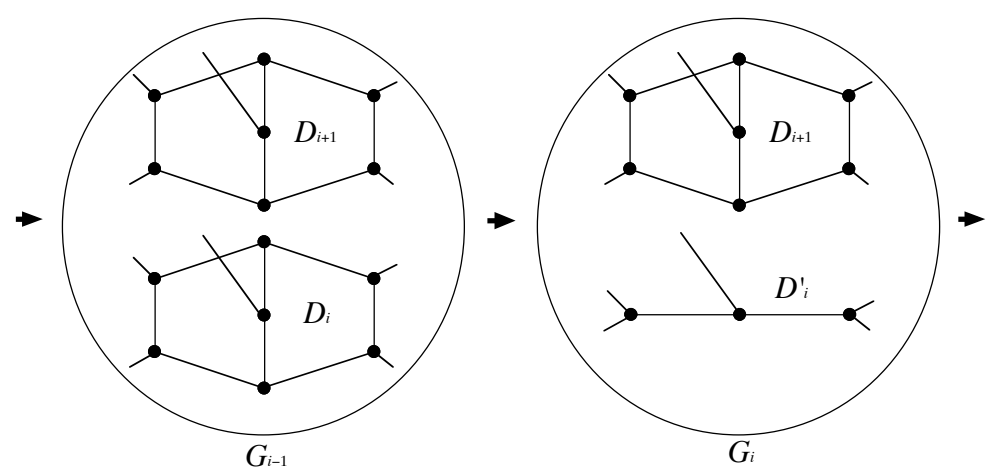

FIG. 7. 
be a maximal set of mutually disjoint 1-cells in $G-\bigcup_{1 \leq l \leq p} D_{l}$ such that $D_{i+1}$ is bad in $G_{i}$ for each $p \leq i \leq p+q-1$, where $G_{i}=G_{i-1} \mid D_{i}$ for $p+1 \leq i \leq p+q$. If there is no bad 1-cell in $G-\bigcup_{1 \leq l \leq p} D_{l}$, then we define $\mathcal{D}_{2}=\emptyset$ and $q=0$. The subgraph in $G_{i}$ corresponding to $D_{i}$ is denoted by $D_{i}^{\prime}$. In this case also, $G-\bigcup_{l \leq i} D_{l}=G_{i}-\bigcup_{l \leq i} D_{l}^{\prime}$ and, by Fact 2.2, each $G_{i}$ is 3-edge-connected for any $0 \leq i \leq p+q$.

Claim 3.2. There is no 1-cell in $G-\bigcup_{l \leq p+q} D_{l}=G_{p+q}-\bigcup_{l \leq p+q} D_{l}^{\prime}$ which is bad in $G_{p+q}$ and there is no 2-cell $C$ in $G_{p+j}-\bigcup_{i \leq p+j} D_{i}^{\prime}$ which is bad in $G_{p+j}$ for any $0 \leq j \leq q$.

Proof. By the maximality of $\mathcal{D}_{2}$, we have the first statement. If there is $1 \leq j \leq q$ such that $G_{p+j}-\bigcup_{i \leq p+j} D_{i}^{\prime}$ contains a 2-cell $C$ which is bad in $G_{p+j}$, then obviously $C$ is bad in $G_{p+j-1}$ also, and so $C$ is bad in $G_{p}$. This contradicts Claim 3.1.

Let $\mathcal{D}_{0}$ be a maximal set of mutually disjoint bad 5-cycles in $G-\bigcup_{i<p+q} D_{i}$. For $\mathcal{D}_{1} \cup \mathcal{D}_{2} \cup \mathcal{D}_{0}$, we define a vertex subset $R^{*}$ of $G$, whose vertices may not be contained in a dominating even subgraph of $G$ which is constructed later.

First, for each cell $D_{i}$ in $\mathcal{D}_{1} \cup \mathcal{D}_{2} \cup \mathcal{D}_{0}$, we define pairs of vertices in $D_{i}$.

1. Let $D_{i}$ be a 2 -cell in $\mathcal{D}_{1}$ and $u_{1} u_{2} u_{3} u_{4} u_{5} u_{6} u_{1}$ be the 6 -cycle in $D_{i}$. See Figure 3(a). The pairs of $D_{i}$ are $\left\{u_{1}, u_{3}\right\}$ and $\left\{u_{4}, u_{6}\right\}$.

2. Let $D_{i}$ be a 1-cell in $\mathcal{D}_{2}$ and $u_{1} u_{2} \cdots u_{8} u_{1}$ be the 8-cycle in $D_{i}$. See Figure 3(b). We define the pair of $D_{i}$ by $\left\{u_{8}, u_{4}\right\}$.

3. For a 5 -cycle $D_{i}=u_{1} u_{2} \cdots u_{5} u_{1}$ in $\mathcal{D}_{0}$, the pair is defined by arbitrary two adjacent vertices in $D_{i}$, e.g., $\left\{u_{1}, u_{2}\right\}$. See Figure $3(\mathrm{c})$.

Let $\mathcal{P}_{0}$ be the set of all the pairs for all $D_{l} \in \mathcal{D}_{1} \cup \mathcal{D}_{2} \cup \mathcal{D}_{0}$. For each pair $\left\{u_{i}, u_{j}\right\} \in \mathcal{P}_{0}$, let $E_{u_{i}, u_{j}}=\partial\left(\left\{u_{i}, u_{j}\right\}\right) \cap \partial D_{l}$, where $\left\{u_{i}, u_{j}\right\} \subset D_{l} \in \mathcal{D}_{1} \cup \mathcal{D}_{2} \cup \mathcal{D}_{0}$. Obviously $0 \leq\left|E_{u_{i}, u_{j}}\right| \leq 2$. Let

$$
\mathcal{P}=\left\{\left\{u_{i}, u_{j}\right\} \in \mathcal{P}_{0}:\left|E_{u_{i}, u_{j}}\right|=2\right\} \text { and } \mathcal{Q}=\bigcup_{\left\{u_{i}, u_{j}\right\} \in \mathcal{P}} E_{u_{i}, u_{j}} .
$$

We define a bipartite graph $H$ on the partite sets $\mathcal{P}$ and $\mathcal{Q}$ by defining the adjacency relation so that $\left\{u_{i}, u_{j}\right\} \in \mathcal{P}$ and $e \in \mathcal{Q}$ are adjacent if and only if $e \in E_{u_{i}, u_{j}}$. Since each element in $\mathcal{Q}$ is adjacent to at most two pairs in $\mathcal{P}$, for any $\mathcal{S} \subset \mathcal{P}$,

$$
2|\mathcal{S}|=\left|E_{H}(\mathcal{S}, N(\mathcal{S}))\right| \leq\left|E_{H}(N(\mathcal{S}), \mathcal{P})\right| \leq 2|N(\mathcal{S})| .
$$

Thus by Hall's theorem, there is a matching $M$ in $H$ covering $\mathcal{P}$. Let $\varphi: \mathcal{P} \rightarrow \mathcal{Q}$ be the injection defined by $M$, i.e., for each $\left\{u_{i}, u_{j}\right\} \in \mathcal{P}$, the pair is adjacent to $\varphi\left(\left\{u_{i}, u_{j}\right\}\right) \in \mathcal{Q}$ by $M$. Let

$R^{*}=\left\{u_{k}: u_{k} \in\left\{u_{i}, u_{j}\right\}\right.$ is the end of the edge $\varphi\left(\left\{u_{i}, u_{j}\right\}\right)$ for some $\left.\left\{u_{i}, u_{j}\right\} \in \mathcal{P}\right\}$.

Notice that there are no edges connecting two vertices in $R^{*}$, except for those connecting $u_{1}$ and $u_{6}$ or connecting $u_{3}$ and $u_{4}$ for some 2 -cell in $\mathcal{D}_{1}$.

Let

$$
\mathcal{D}_{3}=\left\{D_{p+q+1}, D_{p+q+2}, \ldots, D_{p+q+r}\right\} \subset \mathcal{D}_{0}
$$

be a maximal subset of $\mathcal{D}_{0}$ such that for $p+q \leq i \leq p+q+r-1$,

1. $D_{i+1}$ is bad in $G_{i}$, where $u_{i} \in R^{*} \cap D_{i}$, and $G_{i}=\left.G_{i-1}\right|_{u_{i}} D_{i}$, and

2. $D_{i+1}$ intersects neither the 3 -cycle nor the 4-cycle in $G_{i}$.

If there is no such 5-cycle, then we define $\mathcal{D}_{3}=\emptyset$ and $r=0$. We denote by $D_{i}^{\prime}$ the subgraph in $G_{i}$ corresponding to $D_{i} \in \mathcal{D}_{3}$. Notice that each $D_{i}$ has no chord since $D_{i}$ is bad in $G_{i-1}$ and each $G_{i}$ is 3 -edge-connected for any $p+q \leq i \leq p+q+r$ by Fact 2.2. 
Claim 3.3. If there exist $0 \leq j \leq r-1$ and $i \in\{2,1,0\}$ such that $G_{p+q+j}-$ $\bigcup_{l \leq p+q+j} D_{l}^{\prime}-D_{p+q+j+1}$ contains an $i$-cell $C$ which is good in $G_{p+q+j}$, then $C$ is good in $G_{p+q+j+1}$ also.

Proof. Since $C$ is good in $G_{p+q+j}$, there exist an essential 3-cut $T$ of $G_{p+q+j}$ and a 5-cycle $C_{1}$ in $C$ such that $\left|T \cap \partial C_{1}\right| \geq 2$. Let $H_{1}$ and $H_{2}$ be the two components in $G_{p+q+j}-T$. Since $C \subset G_{p+q+j}-\bigcup_{i \leq p+q+j} D_{i}^{\prime}-D_{p+q+j+1}$, the 5-cycle $D_{p+q+j+1}$ is contained in $H_{1}-C$ or $H_{2}-C$, and so both $T$ and $C$ exist in $G_{p+q+r+1}$ also. Thus $C$ is good in $G_{p+q+r+1}$.

Claim 3.4.

1. If $G_{p+q+j}-\bigcup_{i \leq p+q+j} D_{i}^{\prime}$ has a bad 5-cycle, then the 5-cycle is bad in $G_{p+q}$ also.

2. There is neither a 2- nor a 1-cell $C$ in $G_{p+q+j}-\bigcup_{i \leq p+q+j} D_{i}^{\prime}$ which is bad in $G_{p+q+j}$ for any $0 \leq j \leq r$.

Proof. Claim 3.3 implies the first statement immediately. By Claim 3.3, if there is $0 \leq j \leq r$ such that $G_{p+q+j}-\bigcup_{i \leq p+q+j} D_{i}^{\prime}$ contains a 2- or 1-cell $C$ which is bad in $G_{p+q+j}$, then $C$ is bad in $G_{p+q+j-1}$, and so $C$ is bad in $G_{p+q}$. This contradicts Claim 3.2.

Let

$$
S_{0}=\emptyset \text { and } S_{i}=\bigcup_{1 \leq l \leq i} D_{l}^{\prime}
$$

for $1 \leq i \leq p+q+r$. We call a vertex in $S_{i}$ a yellow vertex.

We extend $R^{*}$. Let $D_{i} \in \mathcal{D}_{1}$ and $u_{1} u_{2} u_{3} u_{4} u_{5} u_{6} u_{1}$ be the 6 -cycle. See Figure 3(a). We define $R\left(D_{i}\right)=\left\{u_{2}, u_{5}\right\}$. Let $D_{i} \in \mathcal{D}_{2}$ and $u_{1} u_{2} u_{3} u_{4} u_{5} u_{6} u_{7} u_{8} u_{1}$ be the 8-cycle. See Figure 3(b). We define $R\left(D_{i}\right)=\left\{u_{2}, u_{6}\right\}$. Let

$$
R_{0}=R^{*} \cup \bigcup_{1 \leq l \leq p+q} R\left(D_{l}\right)(\subset V(G))
$$

and

$$
R_{i}=R_{0}-\bigcup_{1 \leq l \leq i} V\left(D_{l}\right)\left(\subset V\left(G_{i}\right)\right)
$$

for $1 \leq i \leq p+q+r$. We call a vertex in $R_{i}$ a red vertex. By the definition of $S_{i}$ and $R_{i}$, there is no vertex in $G_{i}$ which is both red and yellow. Notice that

$$
R_{i}= \begin{cases}R_{i+1} \cup\left(R^{*} \cap D_{i+1}\right) \cup R\left(D_{i+1}\right) & \text { for } 0 \leq i \leq p+q-1, \\ R_{i+1} \cup\left(R^{*} \cap D_{i+1}\right) & \text { for } p+q \leq i \leq p+q+r-1 .\end{cases}
$$

For Theorem 1.1, it is enough to show that for all $0 \leq i \leq p+q+r, G_{i}$ has an even subgraph $F_{i}$ such that the following hold:

1. $F_{i}$ intersects all essential 3 -cuts in $G_{i}$.

2. Every component of $F_{i}$ contains at least five vertices. Especially if a 5-cycle $C$ is a component of $F_{i}$, then $C$ contains a yellow vertex, i.e., $C \cap S_{i} \neq \emptyset$.

3. Every vertex in $G_{i}-F_{i}$ is red, i.e., $G_{i}-F_{i} \subset R_{i}$, and $\left|D_{l}-F_{i}\right| \leq 1$ for $i+1 \leq l \leq p+q+r$.

Indeed by the second condition, any component in $F_{0}$ contains at least six vertices as $S_{0}=\emptyset$. Although $\left(R^{*} \cap V\left(D_{i}\right)\right) \cup R\left(D_{i}\right) \subset R_{0}(1 \leq i \leq p)$ and $R\left(D_{i}\right) \subset R_{0}$ $(p+1 \leq i \leq p+q)$ may not be independent, since $\left|D_{l}-F_{0}\right| \leq 1$ for all $1 \leq l \leq p+q+r$, 
we have $G_{0}-F_{0}\left(\subset R_{0}\right)$ is independent, i.e., $F_{0}$ is dominating $G=G_{0}$. Therefore $F_{0}$ is a desired even subgraph.

We construct $F_{i}$ inductively. First we show the existence of $F_{p+q+r}$. Notice that $G_{i}$ is 3-edge-connected for every $0 \leq i \leq p+q+r$ by Fact 2.2 as we reduced bad cells.

Claim 3.5. There is a 2-factor $F_{p+q+r}$ in $G_{p+q+r}$ such that

1. $F_{p+q+r}$ intersects all 3 -cuts and 4 -cuts in $G_{p+q+r}$ and

2. each 5-cycle $C$ in $F_{p+q+r}$ contains a yellow vertex, i.e., $C \cap S_{p+q+r} \neq \emptyset$.

Proof. Since $G_{p+q+r}$ is a 3 -edge-connected cubic graph, by Corollary C, $G_{p+q+r}$ has a 2 -factor $F_{p+q+r}$ which intersects all 3 -cuts and 4 -cuts. We choose $F_{p+q+r}$ such that the number of components is as small as possible.

Suppose $F_{p+q+r}$ contains a 5 -cycle $C$ without a yellow vertex. If $C$ is good in $G_{p+q+r}$, then there is an essential 3-cut $T$ such that $|T \cap \partial C| \geq 2$, and $F_{p+q+r}$ does not intersect the 3-cut $T$, a contradiction. Therefore $C$ is bad in $G_{p+q+r}$. Since $C$ has no yellow vertex, $C \subset G_{p+q+r}-S_{p+q+r}$, and so the 5-cycle $C$ exists in $G_{p+q}$ and, by Claim 3.4, $C$ is bad in $G_{p+q}$ also.

Suppose $C \notin \mathcal{D}_{0}$. By the maximality of $\mathcal{D}_{0}$, there is a bad 5-cycle $D \in \mathcal{D}_{0}$ intersecting $C$. If $|E(C \cap D)| \leq 2$, then $C \cup D$ is a 2- or 1-cell in $G_{p+q}$. Since both $C$ and $D$ are bad in $G_{p+q}, C \cup D$ is bad in $G_{p+q}$. This contradicts Claim 3.2.

If $|E(C \cap D)|=3$, then $D$ is a 5 -cycle in $G_{p+q+r}$ also. However, $F_{p+q+r}$ does not contain the vertex in $D-C$ as $C$ is a component of $F_{p+q+r}$. This is a contradiction.

Therefore $C \in \mathcal{D}_{0}$. Since $C$ is bad in $G_{p+q+r}$ and $C \notin \mathcal{D}_{3}, C$ intersects a 3 - or a 4-cycle $C_{1}$ in $G_{p+q+r}$. Since $C$ is bad, $C$ has no chord, and so $C_{1}-C \neq \emptyset$. If $C_{1}-C$ is a vertex $w$, then $w$ is not contained in $F_{p+q+r}$ as $C$ is a component of the 2 -factor $F_{p+q+r}$. This is a contradiction.

If $C_{1}-C$ contains an edge $w w^{\prime}$, then there is a component $C_{2}$ in $F_{p+q+r}$ containing the edge $w w^{\prime}$. Since the symmetric difference $\widetilde{C}=C \triangle C_{1} \triangle C_{2}$ is a cycle, the subgraph $\left(F_{p+q+r}-C \cup C_{2}\right) \cup \widetilde{C}$ is a 2 -factor of $G_{p+q+r}$ in which the number of components is less than $F_{p+q+r}$. This contradicts the choice of $F_{p+q+r}$. Thus $C$ contains a yellow vertex.

Suppose $G_{i+1}$ has a desired even subgraph $F_{i+1}$ for $1 \leq i+1 \leq p+q+r$. Since $G_{i+1}$ has no vertex which is both yellow and red and $F_{i+1}$ contains every vertex which is not red in $G_{i+1}$, we have $\bigcup_{l \leq i+1} D_{l}^{\prime} \subset F_{i+1}$.

CLAIM 3.6. If $F_{i}$ is an even subgraph of $G_{i}$ obtained from $F_{i+1}$ by replacing edges in $D_{i+1}^{\prime}$ with edges in $D_{i+1}$, i.e.,

$$
E\left(F_{i+1}\right)-E\left(D_{i+1}^{\prime}\right)=E\left(F_{i}\right)-E\left(D_{i+1}\right),
$$

then the following holds:

1. $F_{i}$ intersects all essential 3 -cuts in $G_{i}$.

2. Every component $C$ of $F_{i}$ intersecting no edge of $D_{i+1}$ contains at least five vertices. Especially if $C$ is a 5 -cycle, then $C$ contains a yellow vertex, i.e., $C \cap S_{i} \neq \emptyset$.

3. Every vertex in $G_{i}-F_{i}-D_{i+1}$ is red, i.e., $G_{i}-F_{i}-D_{i+1} \subset R_{i}$, and $\left|D_{j}-F_{i}\right| \leq$ 1 for $j \geq i+2$.

Proof.

1. Let $T$ be any essential 3-cut of $G_{i}$. Since $D_{i+1}$ is bad in $G_{i}, T \cap E\left(D_{i+1}\right)=\emptyset$ by Lemma 2.3. This implies $T$ is an essential 3-cut of $G_{i+1}$ and $T \cap E\left(D_{i+1}^{\prime}\right)=\emptyset$. Thus $F_{i+1}-E\left(D_{i+1}^{\prime}\right)=F_{i}-E\left(D_{i+1}\right)$ intersects $T$. 
2. Obviously $C$ is a component of $F_{i+1}$ also. Thus $|C| \geq 5$ and $C$ contains a yellow vertex in $S_{i+1}-V\left(D_{i+1}^{\prime}\right)=S_{i}$ if $|C|=5$.

3. Since $G_{i}-D_{i+1}=G_{i+1}-D_{i+1}^{\prime}$, we have $G_{i}-D_{i+1}-F_{i}=G_{i+1}-D_{i+1}^{\prime}-F_{i+1} \subset$ $R_{i+1} \subset R_{i}$ by (1). For $j \geq i+2$,

$$
D_{j}-F_{i}=D_{j}-F_{i+1},
$$

and so we have $\left|D_{j}-F_{i}\right|=\left|D_{j}-F_{i+1}\right| \leq 1$.

In the remaining part of this paper, we will construct a desired even subgraph $F_{i}$ of $G_{i}$ from $F_{i+1}$ by replacing edges in $D_{i+1}^{\prime}$ with edges in $D_{i+1}$. From the above claim, it is enough to show that $F_{i}$ satisfies the following:

A1. Every component $C$ containing an edge of $D_{i+1}$ in $F_{i}$ contains at least five vertices. Especially if $C$ is a 5 -cycle, then $C$ contains a yellow vertex, i.e., $C \cap S_{i} \neq \emptyset$.

A2. A vertex in $D_{i+1}-F_{i}$ is red, i.e., $D_{i+1}-F_{i} \subset R_{i}$, and $\left|D_{i+1}-F_{i}\right| \leq 1$.

We divide our argument into the following three cases:

1. $0 \leq i \leq p-1$.

2. $p+q \leq i \leq p+q+r-1$.

3. $p \leq i \leq p+q-1$.

The first case is easier than the other cases. If there is a vertex in $G_{i}-D_{i+1}$ which is adjacent to $u \in D_{i+1}$, then we denote the vertex by $u^{\prime}$.

1. $0 \leq i \leq p-1$, i.e., $D_{i+1} \in \mathcal{D}_{1}$.

Since $V\left(D_{i+1}^{\prime}\right) \subset F_{i+1}$ and $F_{i+1}$ is an even subgraph, $\left|F_{i+1} \cap \partial D_{i+1}^{\prime}\right|$ is 2 or 4 . If the subgraph induced by $V\left(D_{i+1}\right)$ contains an edge that is not in $E\left(D_{i+1}\right)$, then $\left|\partial D_{i+1}\right|=3$. This implies $D_{i+1}$ contains a good 5 -cycle, i.e., $D_{i+1}$ is good in $G_{i}$. This contradicts our assumption. Therefore, both $\left\{u_{1}, u_{3}\right\}$ and $\left\{u_{4}, u_{6}\right\}$ contain a red vertex.

Case 1. $\left|F_{i+1} \cap \partial D_{i+1}^{\prime}\right|=4$.

Since $D_{i+1}^{\prime} \subset F_{i+1}$, by symmetry, we may suppose

$$
F_{i+1} \cap \partial D_{i+1}^{\prime}=\left\{u_{1} u_{6}^{\prime}, u_{1} u_{1}^{\prime}, u_{2} w^{\prime}, u_{3} u_{3}^{\prime}\right\}
$$

and then $F_{i+1}$ contains the edge $u_{2} u_{3}$. See Figure 8 . Let $F_{i}$ be the even subgraph obtained from $F_{i+1}$ by replacing

$$
u_{1}^{\prime} u_{1} u_{6}^{\prime} \text { and } w^{\prime} u_{2} u_{3} u_{3}^{\prime} \text { by } u_{1}^{\prime} u_{1} u_{6} u_{6}^{\prime} \text { and } w^{\prime} w u_{5} u_{4} u_{3} u_{3}^{\prime} .
$$

Since every component $C$ containing an edge of $D_{i+1}$ in $F_{i}$ contains at least six vertices, A1 holds. Since $u_{2} \in R\left(D_{i+1}\right) \subset R_{i}$, A2 holds.

Case 2. $\left|F_{i+1} \cap \partial D_{i+1}^{\prime}\right|=2$.

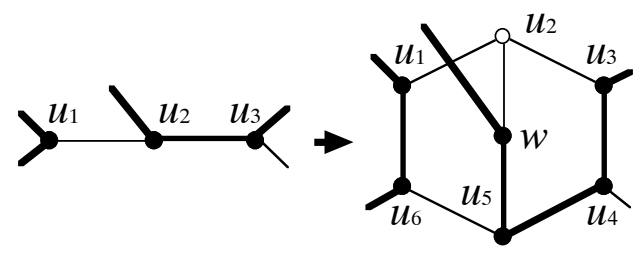

FIG. 8. 


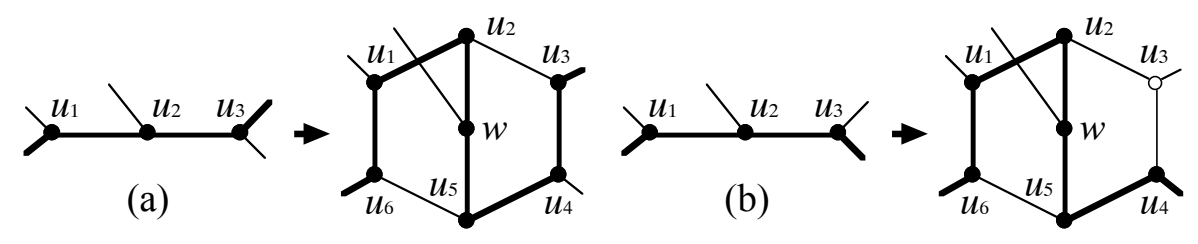

FIG. 9.

Since $D_{i+1}^{\prime} \subset F_{i+1}, F_{i+1} \cap \partial D_{i+1}^{\prime}$ does not contain the edge $u_{2} w^{\prime}$. Thus by symmetry, we may suppose

$$
F_{i+1} \cap \partial D_{i+1}^{\prime} \text { is }\left\{u_{1} u_{6}^{\prime}, u_{3} u_{3}^{\prime}\right\} \text { or }\left\{u_{1} u_{6}^{\prime}, u_{3} u_{4}^{\prime}\right\} .
$$

See Figure 9. If the intersection is $\left\{u_{1} u_{6}^{\prime}, u_{3} u_{3}^{\prime}\right\}$, then the even subgraph $F_{i}$ obtained from $F_{i+1}$ by replacing

$$
u_{1} u_{2} u_{3} \text { by } u_{6} u_{1} u_{2} w u_{5} u_{4} u_{3}
$$

is a desired even subgraph because both $\mathrm{A} 1$ and A2 hold as $V\left(D_{i+1}\right) \subset F_{i}$.

Suppose $F_{i+1} \cap \partial D_{i+1}^{\prime}=\left\{u_{1} u_{6}^{\prime}, u_{3} u_{4}^{\prime}\right\}$. For the pair $\left\{u_{1}, u_{3}\right\}$, if $u_{3}$ is red, i.e., $u_{3} \in R_{i}$, then the even subgraph $F_{i}$ obtained from $F_{i+1}$ by replacing

$$
u_{6}^{\prime} u_{1} u_{2} u_{3} u_{3}^{\prime} \text { by } u_{6}^{\prime} u_{6} u_{1} u_{2} w u_{5} u_{4} u_{4}^{\prime}
$$

is a desired even subgraph because both A1 and A2 hold. Similarly if $u_{1} \in R_{i}$, then the even subgraph $F_{i}$ obtained from $F_{i+1}$ by replacing

$$
u_{6}^{\prime} u_{1} u_{2} u_{3} u_{4}^{\prime} \text { by } u_{6}^{\prime} u_{6} u_{5} w u_{2} u_{3} u_{4} u_{4}^{\prime}
$$

is a desired even subgraph.

2. $p+q \leq i \leq p+q+r-1$, i.e., $D_{i+1} \in \mathcal{D}_{3}$.

In this case, $D_{i+1}=u_{1} u_{2} \cdots u_{5} u_{1}$ is a 5 -cycle. By symmetry, we may suppose $u_{2}$ is red, i.e., $u_{2} \in R_{i}$. Since $V\left(D_{i+1}^{\prime}\right) \subset F_{i+1},\left|F_{i+1} \cap \partial D_{i+1}^{\prime}\right|$ is 2 or 4 .

Case 1. $\left|F_{i+1} \cap \partial D_{i+1}^{\prime}\right|=2$.

Notice that $F_{i+1} \cap \partial D_{i+1}^{\prime}$ does not contain $u_{2} u_{2}^{\prime}$ because $V\left(D_{i+1}^{\prime}\right) \subset F_{i+1}$. Hence by symmetry, we have the following three cases:

$$
F_{i+1} \cap \partial D_{i+1}^{\prime} \text { is }\left\{u_{1} u_{4}^{\prime}, u_{3} u_{5}^{\prime}\right\},\left\{u_{1} u_{4}^{\prime}, u_{3} u_{3}^{\prime}\right\} \text { or }\left\{u_{1} u_{1}^{\prime}, u_{3} u_{3}^{\prime}\right\} .
$$

(i) Suppose $F_{i+1} \cap \partial D_{i+1}^{\prime}=\left\{u_{1} u_{4}^{\prime}, u_{3} u_{5}^{\prime}\right\}$. See Figure 10(a). As $V\left(D_{i+1}^{\prime}\right) \subset F_{i+1}$, $F_{i+1}$ contains the path $u_{4}^{\prime} u_{1} u_{2} u_{3} u_{5}^{\prime}$. Let $F_{i}$ be the even subgraph in $G_{i}$ which is obtained from $F_{i+1}$ by replacing

$$
u_{4}^{\prime} u_{1} u_{2} u_{3} u_{5}^{\prime} \text { by } u_{4}^{\prime} u_{4} u_{3} u_{2} u_{1} u_{5} u_{5}^{\prime}
$$

Obviously A1 holds. Since $V\left(D_{i+1}\right) \subset F_{i}$, A2 holds.

(ii) Suppose $F_{i+1} \cap \partial D_{i+1}^{\prime}=\left\{u_{1} u_{4}^{\prime}, u_{3} u_{3}^{\prime}\right\}$. See Figure 10(b). Then $F_{i+1}$ contains the path $u_{4}^{\prime} u_{1} u_{2} u_{3} u_{3}^{\prime}$ as $V\left(D_{i+1}^{\prime}\right) \subset F_{i+1}$. Let $F_{i}$ be the even subgraph in $G_{i}$ which is obtained from $F_{i+1}$ by replacing

$$
u_{4}^{\prime} u_{1} u_{2} u_{3} u_{3}^{\prime} \text { by } u_{4}^{\prime} u_{4} u_{5} u_{1} u_{2} u_{3} u_{3}^{\prime} \text {. }
$$

Obviously both A1 and A2 hold.

(iii) Suppose $F_{i+1} \cap \partial D_{i+1}^{\prime}=\left\{u_{1} u_{1}^{\prime}, u_{3} u_{3}^{\prime}\right\}$. See Figure 10(c). Let $F_{i}$ be the even subgraph in $G_{i}$ which is obtained from $F_{i+1}$ by replacing 
(a)

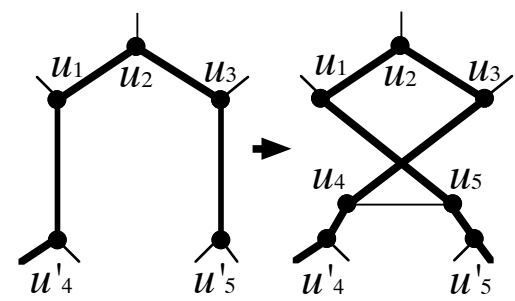

(b)

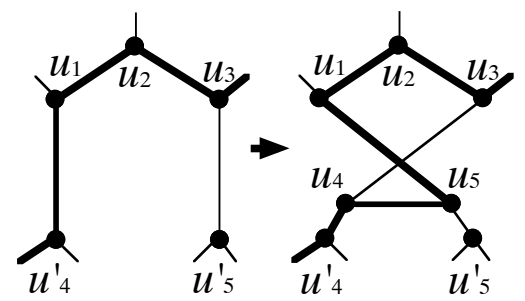

(c)

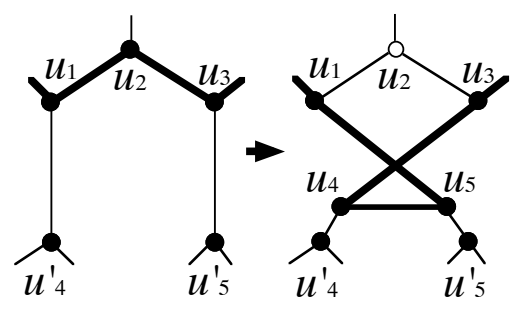

FIG. 10

(a)

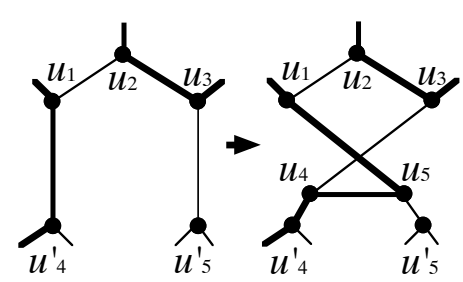

(b)

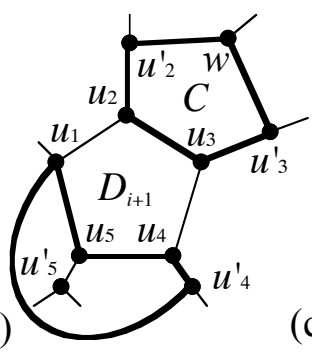

(c)

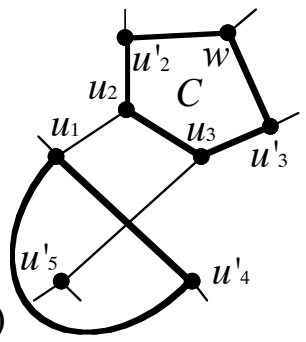

FIG. 11

$$
u_{1}^{\prime} u_{1} u_{2} u_{3} u_{3}^{\prime} \text { by } u_{1}^{\prime} u_{1} u_{5} u_{4} u_{3} u_{3}^{\prime} .
$$

Since the component in $F_{i}$ containing an edge in $D_{i+1}$ contains at least six vertices, A1 holds. As $D_{i+1}-F_{i}=\left\{u_{2}\right\} \subset R_{i}$, A2 holds.

Case 2. $\left|F_{i+1} \cap \partial D_{i+1}^{\prime}\right|=4$.

As $V\left(D_{i+1}^{\prime}\right) \subset F_{i+1}, F_{i+1} \cap \partial D_{i+1}^{\prime}$ is not $\left\{u_{1} u_{4}^{\prime}, u_{1} u_{1}^{\prime}, u_{3} u_{3}^{\prime}, u_{3} u_{5}^{\prime}\right\}$. Thus by symmetry, we have two cases.

(i) Suppose

$$
F_{i+1} \cap \partial D_{i+1}^{\prime}=\left\{u_{1} u_{4}^{\prime}, u_{1} u_{1}^{\prime}, u_{2} u_{2}^{\prime}, u_{3} u_{3}^{\prime}\right\},
$$

and then $u_{2} u_{3} \in F_{i+1}$. See Figure 11(a). Let $F_{i}$ be the even subgraph in $G_{i}$ which is obtained from $F_{i+1}$ by replacing

$$
u_{4}^{\prime} u_{1} u_{1}^{\prime} \text { by } u_{4}^{\prime} u_{4} u_{5} u_{1} u_{1}^{\prime} .
$$

Since $V\left(D_{i+1}\right) \subset F_{i}$, A2 holds.

The component continuing $u_{4}^{\prime} u_{4} u_{5} u_{1} u_{1}^{\prime}$ of $F_{i}$ contains at least six vertices. Suppose $C=u_{2} u_{3} u_{3}^{\prime} w u_{2}^{\prime} u_{2}$ is a 5-cycle and $C \cap S_{i}=\emptyset$. See Figure 11(b). Then $\widetilde{C}=C \cup D_{i+1}$ is a 1 -cell in $G_{i}-\bigcup_{j<i} D_{j}^{\prime}$. Since $D_{i+1} \in \mathcal{D}_{3}, D_{i+1}$ is bad in $G_{i}$. Suppose that $C$ is good and let $\bar{T}$ be an essential 3-cut such that 
(a)

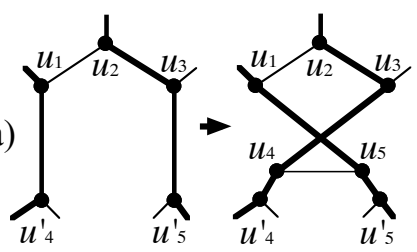

(b)

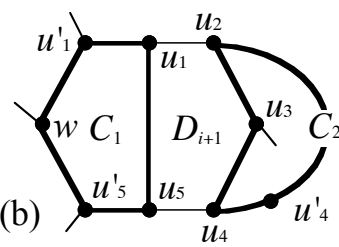

(d)

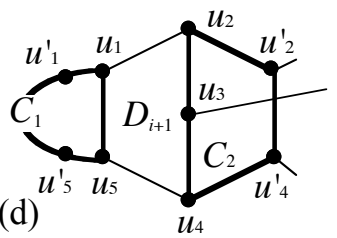

(c)

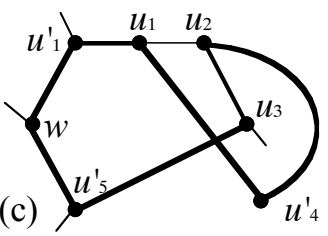

(e)

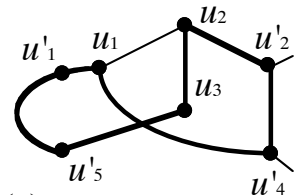

FIG. 12 .

$|T \cap \partial C| \geq 2$. Since $D_{i+1}$ is bad, $T \cap \partial C \subset \partial C-\left\{u_{2} u_{1}, u_{3} u_{4}\right\}$ by Lemma 2.3 . Since $T$ is an essential 3-cut of $G_{i+1}$ also and $F_{i+1}$ contains $C$ as a component, $F_{i+1}$ does not intersect $T$, a contradiction. See Figure 11(c). Thus both $D_{i+1}$ and $C$ are bad, and so $\widetilde{C}$ is a bad 1-cell in $G_{i}$. This contradicts Claim 3.4.

(ii) Suppose

$$
F_{i+1} \cap \partial D_{i+1}^{\prime}=\left\{u_{1} u_{4}^{\prime}, u_{1} u_{1}^{\prime}, u_{2} u_{2}^{\prime}, u_{3} u_{5}^{\prime}\right\},
$$

and then $u_{2} u_{3} \in F_{i+1}$. See Figure 12(a).

Let $F_{i}$ be the even subgraph in $G_{i}$ which is obtained from $F_{i+1}$ by replacing

$$
u_{4}^{\prime} u_{1} u_{1}^{\prime} \text { and } u_{2}^{\prime} u_{2} u_{3} u_{5}^{\prime} \text { by } u_{4}^{\prime} u_{4} u_{3} u_{2} u_{2}^{\prime} \text { and } u_{1}^{\prime} u_{1} u_{5} u_{5}^{\prime} \text {. }
$$

Since $V\left(D_{i+1}\right) \subset F_{i}$, A2 holds.

Let $C_{1}$ and $C_{2}$ be the components in $F_{i}$ containing $u_{1} u_{5}$ and $u_{2} u_{3} u_{4}$, respectively. Suppose $C_{1}$ or $C_{2}$ contains at most five vertices, and then $C_{1} \neq C_{2}$. Since $D_{i+1}$ intersects neither the 3 -cycle nor the 4 -cycle, $C_{1}$ or $C_{2}$ is a 5 -cycle.

Suppose $C_{1}=u_{1} u_{5} u_{5}^{\prime} w u_{1}^{\prime} u_{1}$ is a 5 -cycle and $C_{1} \cap S_{i}=\emptyset$. See Figure 12(b). Then $\widetilde{C_{1}}=C_{1} \cup D_{i+1}$ is a 1-cell in $G_{i}$. By Claim 3.4, $\widetilde{C_{1}}$ is good. Since $D_{i+1}$ is bad, $C_{1}$ is good, and so there is an essential 3-cut $T$ in $G_{i}$ such that $\left|T \cap \partial C_{1}\right| \geq 2$, and by Lemma $2.3 T \cap \partial C_{1} \subset \partial C_{1}-\left\{u_{1} u_{2}, u_{5} u_{4}\right\}$. Thus $T$ is an essential 3-cut of $G_{i+1}$. Since $F_{i+1}$ contains the path $u_{1} u_{1}^{\prime} w u_{5}^{\prime} u_{3}, F_{i+1}$ does not intersect $T$, a contradiction. See Figure 12(c).

Suppose $C_{2}=u_{2} u_{3} u_{4} u_{4}^{\prime} u_{2}^{\prime} u_{2}$ is a 5 -cycle and $C_{2} \cap S_{i}=\emptyset$. See Figure 12(d). Then $\widetilde{C_{2}}=C_{2} \cup D_{i+1}$ is a 2-cell in $G_{i}$. By Claim 3.4, $\widetilde{C_{2}}$ is good, and so, as in the above case, $G_{i}$ has an essential 3-cut $T$ such that $\left|T \cap \partial C_{2}\right| \geq 2$, and $T \cap \partial C_{2} \subset \partial C_{2}-\left\{u_{1} u_{2}, u_{4} u_{5}\right\}$. Hence $T$ is an essential 3 -cut of $G_{i+1}$. Since $F_{i+1}$ contains the path $u_{5}^{\prime} u_{3} u_{2} u_{2}^{\prime} u_{4}^{\prime} u_{1}$, $F_{i+1}$ does not intersect $T$, a contradiction. See Figure 12(e).

3. $p \leq i \leq p+q-1$, i.e., $D_{i+1} \in \mathcal{D}_{2}$.

Since $V\left(D_{i+1}^{\prime}\right) \subset F_{i+1},\left|F_{i+1} \cap \partial D_{i+1}^{\prime}\right|$ is 0,2 , 4, or 6 .

Case 1. $\left|F_{i+1} \cap \partial D_{i+1}^{\prime}\right|=0$.

In this case, the 6 -cycle $u_{1} u_{3} u_{4} u_{5} u_{7} u_{8}$ is contained in $F_{i+1}$, and replacing it with the 8-cycle $u_{1} u_{2} u_{3} u_{4} u_{5} u_{6} u_{7} u_{8}$, we obtain the even subgraph $F_{i}$ in $G_{i}$. Obviously, both $\mathrm{A} 1$ and $\mathrm{A} 2$ hold.

Case 2. $\left|F_{i+1} \cap \partial D_{i+1}^{\prime}\right|=2$. 

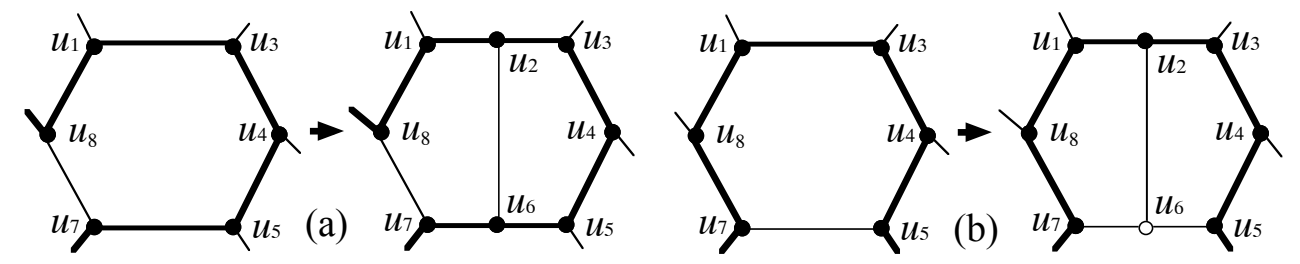

FIG. 13
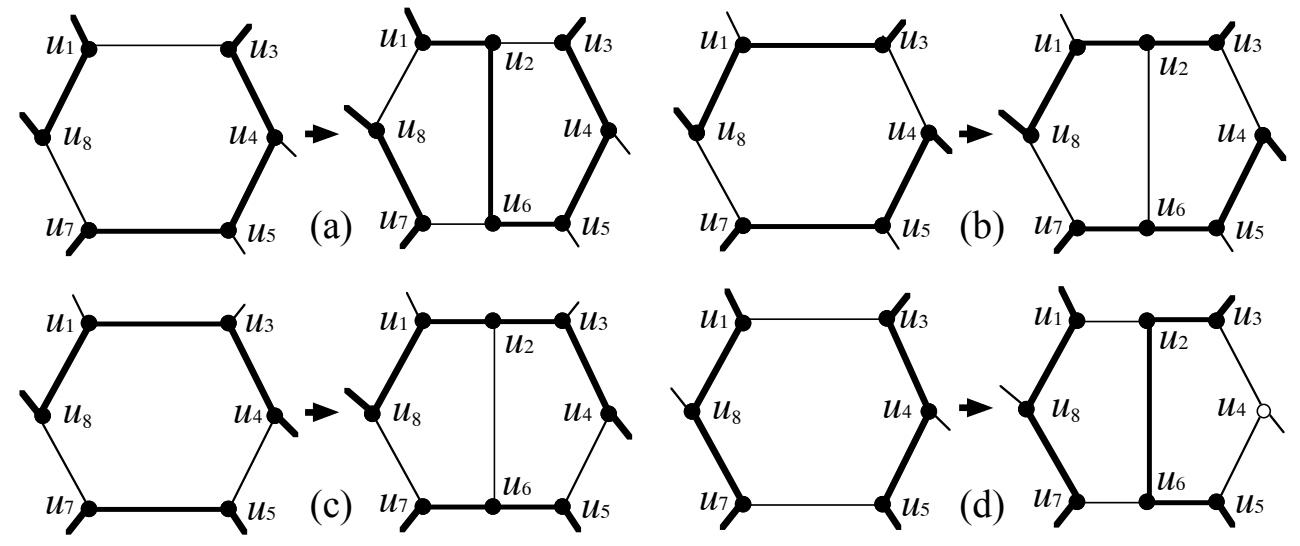

FIG. 14.

Since $V\left(D_{i+1}^{\prime}\right) \subset F_{i+1}$ and $F_{i+1}$ is an even subgraph, by symmetry we have two cases. If $F_{i+1} \cap \partial D_{i+1}^{\prime}=\left\{u_{7} u_{7}^{\prime}, u_{8} u_{8}^{\prime}\right\}$, then $F_{i+1}$ contains the path $u_{8} u_{1} u_{3} u_{4} u_{5} u_{7}$. See Figure 13(a). Then the even subgraph $F_{i}$ obtained from $F_{i+1}$ by replacing

$$
u_{8}^{\prime} u_{8} u_{1} u_{3} u_{4} u_{5} u_{7} u_{7}^{\prime} \text { by } u_{8}^{\prime} u_{8} u_{1} u_{2} u_{3} u_{4} u_{5} u_{6} u_{7} u_{7}^{\prime}
$$

is a desired even subgraph because both A1 and A2 hold. Similarly, we can show the case of $F_{i+1} \cap \partial D_{i+1}^{\prime}=\left\{u_{7} u_{7}^{\prime}, u_{5} u_{5}^{\prime}\right\}$ since $u_{6} \in R\left(D_{i+1}\right) \subset R_{i}$. See Figure 13(b).

Case 3. $\left|F_{i+1} \cap \partial D_{i+1}^{\prime}\right|=4$.

By symmetry, we have four cases:

(i) $F_{i+1} \cap \partial D_{i+1}^{\prime}=\left\{u_{7} u_{7}^{\prime}, u_{8} u_{8}^{\prime}, u_{1} u_{1}^{\prime}, u_{3} u_{3}^{\prime}\right\}$.

Since $V\left(D_{i+1}^{\prime}\right) \subset F_{i+1}, F_{i+1}$ contains the paths $u_{8} u_{1}$ and $u_{3} u_{4} u_{5} u_{7}$. See Figure 14(a). Let $P_{1}$ and $P_{2}$ be the two paths obtained from the cycles in $F_{i+1}$ intersecting $D_{i+1}^{\prime}$ by removing edges in $E\left(D_{i+1}^{\prime}\right)$ and isolated vertices. By symmetry, we may suppose $u_{3} \in P_{1}$. If $u_{7} \in P_{1}$ or $u_{8} \in P_{1}$, then the even subgraph $F_{i}$ obtained from $F_{i+1}$ by replacing

$$
u_{1}^{\prime} u_{1} u_{8} u_{8}^{\prime} \text { and } u_{3}^{\prime} u_{3} u_{4} u_{5} u_{7} u_{7}^{\prime} \text { by } u_{7}^{\prime} u_{7} u_{8} u_{8}^{\prime} \text { and } u_{3}^{\prime} u_{3} u_{4} u_{5} u_{6} u_{2} u_{1} u_{1}^{\prime}
$$

is a desired even subgraph because both A1 and A2 hold. See Figure 14(a). In the case of $u_{1} \in P_{1}$, let $F_{i}$ be the even subgraph obtained from $F_{i+1}$ by replacing

$$
u_{3}^{\prime} u_{3} u_{4} u_{5} u_{7} u_{7}^{\prime} \text { by } u_{3}^{\prime} u_{3} u_{4} u_{5} u_{6} u_{7} u_{7}^{\prime} \text {. }
$$

Obviously A1 holds. Since $u_{2} \in R\left(D_{i+1}\right) \subset R_{i}$, A2 holds. 

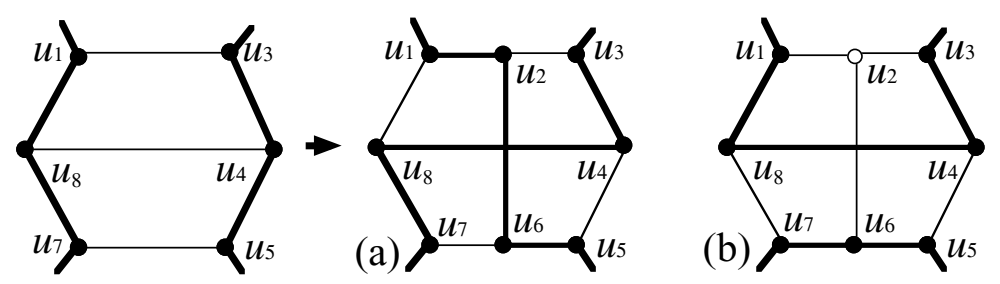

FIG. 15.

(ii) $F_{i+1} \cap \partial D_{i+1}^{\prime}=\left\{u_{7} u_{7}^{\prime}, u_{8} u_{8}^{\prime}, u_{3} u_{3}^{\prime}, u_{4} u_{4}^{\prime}\right\}$.

Then $F_{i+1}$ contains the paths $u_{8} u_{1} u_{3}$ and $u_{4} u_{5} u_{7}$. See Figure 14(b). Then the even subgraph $F_{i}$ obtained from $F_{i+1}$ by replacing

$$
u_{8}^{\prime} u_{8} u_{1} u_{3} u_{3}^{\prime} \text { and } u_{4}^{\prime} u_{4} u_{5} u_{7} u_{7}^{\prime} \text { by } u_{8}^{\prime} u_{8} u_{1} u_{2} u_{3} u_{3}^{\prime} \text { and } u_{4}^{\prime} u_{4} u_{5} u_{6} u_{7} u_{7}^{\prime}
$$

is a desired even subgraph because both A1 and A2 hold.

(iii) Similarly we can show the case that $F_{i+1} \cap \partial D_{i+1}^{\prime}=\left\{u_{7} u_{7}^{\prime}, u_{8} u_{8}^{\prime}, u_{4} u_{4}^{\prime}, u_{5} u_{5}^{\prime}\right\}$. See Figure 14(c).

(iv) $F_{i+1} \cap \partial D_{i+1}^{\prime}=\left\{u_{7} u_{7}^{\prime}, u_{1} u_{1}^{\prime}, u_{3} u_{3}^{\prime}, u_{5} u_{5}^{\prime}\right\}$.

Suppose first that $u_{4} u_{8} \in E(G)$. Then, $F_{i+1}$ contains either the paths $u_{7} u_{8} u_{1}$ and $u_{3} u_{4} u_{5}$, or the paths $u_{1} u_{8} u_{4} u_{3}$ and $u_{5} u_{7}$, or $u_{1} u_{3}$ and $u_{5} u_{4} u_{8} u_{7}$. See Figure 15 for the case of the paths $u_{7} u_{8} u_{1}$ and $u_{3} u_{4} u_{5}$. Let $P_{1}$ and $P_{2}$ be the two paths obtained from the cycles in $F_{i+1}$ intersecting $D_{i+1}^{\prime}$ by removing edges in $E\left(D_{i+1}^{\prime}\right)$ and isolated vertices. By symmetry, we may suppose $u_{3} \in$ $P_{1}$. If $u_{1} \in P_{1}$ or $u_{5} \in P_{1}$, then the even subgraph $F_{i}$ obtained from $F_{i+1}$ by replacing the paths inside $D_{i+1}^{\prime}$

\section{by $u_{7} u_{8} u_{4} u_{3}$ and $u_{1} u_{2} u_{6} u_{5}$}

is a desired even subgraph because both A1 and A2 hold. See Figure 15(a).

In the case of $u_{7} \in P_{1}$, let $F_{i}$ be the even subgraph obtained from $F_{i+1}$ by replacing the paths inside $D_{i+1}^{\prime}$

by $u_{1} u_{8} u_{4} u_{3}$ and $u_{7} u_{6} u_{5}$.

See Figure 15(b). Obviously A1 holds. Since $u_{2} \in R\left(D_{i+1}\right) \subset R_{i}$, A2 holds.

Therefore, we may assume that $u_{4} u_{8} \notin E(G)$. Since there are components in $F_{i+1}$ containing $u_{7} u_{8} u_{1}$ and $u_{3} u_{4} u_{5}$, both $u_{4}^{\prime}$ and $u_{8}^{\prime}$ exist. Thus $\left|E_{u_{4}, u_{8}}\right|=2$, and so one of $u_{4}$ and $u_{8}$ is in $R_{i}$. By symmetry, we may suppose $u_{4} \in R_{i}$. Let $F_{i}$ be the even subgraph obtained from $F_{i+1}$ by replacing

$$
u_{3}^{\prime} u_{3} u_{4} u_{5} u_{5}^{\prime} \text { by } u_{3}^{\prime} u_{3} u_{2} u_{6} u_{5} u_{5}^{\prime} \text {. }
$$

See Figure 14(d). Obviously A2 holds.

Suppose the component $C_{1}$ of $F_{i}$ containing $u_{7} u_{8} u_{1}$ is a 5-cycle and $C_{1} \cap S_{i}=\emptyset$. Let $C_{1}=u_{1} u_{8} u_{7} u_{7}^{\prime} u_{1}^{\prime} u_{1}$ and $C_{2}=u_{1} u_{2} u_{6} u_{7} u_{8} u_{1}$. See Figure 16(a). Then $C=C_{1} \cup C_{2}$ is a 2-cell in $G_{i}$. By Claim 3.2,C is good in $G_{i}$. Since $D_{i+1}$ is bad, $C_{2}$ is bad, and so $C_{1}$ is good. Thus there is an essential 3-cut $T$ such that $\left|T \cap \partial C_{1}\right| \geq 2$. Since $C_{2}$ is bad, $T \cap \partial C_{1} \subset \partial C_{1}-\left\{u_{1} u_{2}, u_{7} u_{6}\right\}$. Hence $T$ is an essential 3-cut of $G_{i+1}$. Since $F_{i+1}$ contains $C_{1}$ as a component, $F_{i+1}$ does not intersect $T$, a contradiction. See Figure 16(b).

Case 4. $\left|F_{i+1} \cap \partial D_{i+1}^{\prime}\right|=6$. 
(a)

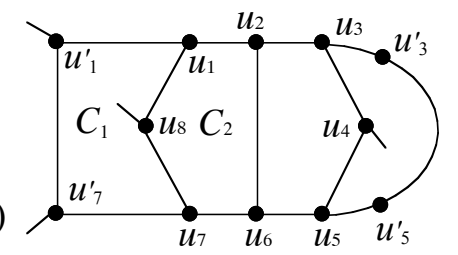

(b)

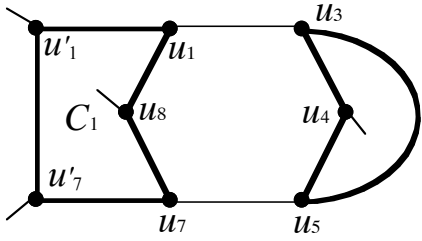

FIG. 16.

(a)
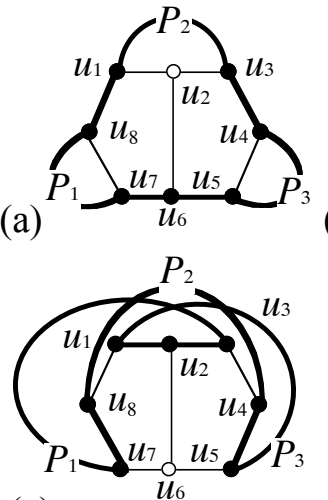

(e) (b)
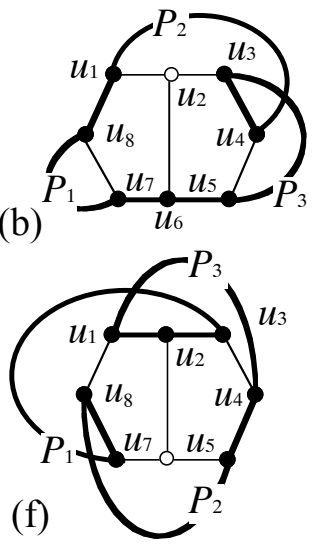

(c)

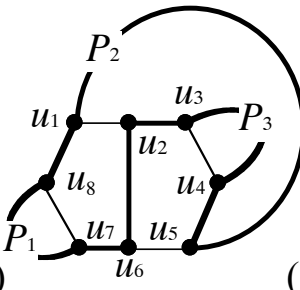

(d)

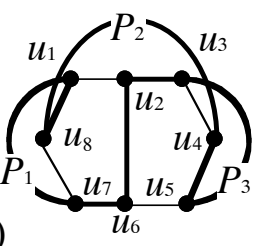

(g)

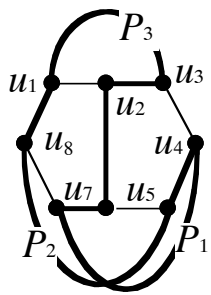

(h)

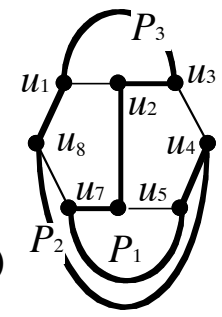

FIG. 17.

In this case, $F_{i+1}$ contains all the edges in $\partial D_{i+1}^{\prime}$. Let $P_{1}, P_{2}$, and $P_{3}$ be the three paths obtained from the cycles in $F_{i+1}$ intersecting $D_{i+1}^{\prime}$ by removing edges in $E\left(D_{i+1}^{\prime}\right)$. By symmetry, we may suppose $u_{7} \in P_{1}$. It is easy to confirm that for all the following cases, both A1 and A2 hold.

(i) The ends of $P_{1}$ are $u_{7}$ and $u_{8}$.

(a) If the ends of $P_{2}$ are $u_{1}$ and $u_{3}$, then let $F_{i}$ be the even subgraph obtained from $F_{i+1}$ by replacing the cycles in $F_{i+1}$ intersecting $D_{i+1}^{\prime}$ with the cycle

$$
u_{7} P_{1} u_{8} u_{1} P_{2} u_{3} u_{4} P_{3} u_{5} u_{6} u_{7} .
$$

See Figure 17(a).

(b) If the ends of $P_{2}$ are $u_{1}$ and $u_{4}$, then let $F_{i}$ be the even subgraph obtained from $F_{i+1}$ by replacing the cycles in $F_{i+1}$ intersecting $D_{i+1}^{\prime}$ with the cycle

$$
u_{7} P_{1} u_{8} u_{1} P_{2} u_{4} u_{3} P_{3} u_{5} u_{6} u_{7} .
$$

See Figure 17(b).

(c) If the ends of $P_{2}$ are $u_{1}$ and $u_{5}$, then let $F_{i}$ be the even subgraph obtained from $F_{i+1}$ by replacing the cycles in $F_{i+1}$ intersecting $D_{i+1}^{\prime}$ with the cycle

$$
u_{7} P_{1} u_{8} u_{1} P_{2} u_{5} u_{4} P_{3} u_{3} u_{2} u_{6} u_{7} .
$$

See Figure 17(c). 
Notice that by symmetry, we finished showing all the cases where there is a path joining $u_{i}$ and $u_{i+1}$ for any $i$ by the case (i).

(ii) The ends of $P_{1}$ are $u_{7}$ and $u_{1}$.

In this case, the ends of $P_{2}$ are $u_{8}$ and $u_{4}$; otherwise there is a path joining $u_{i}$ and $u_{i+1}$ for some $i$. Let $F_{i}$ be the even subgraph obtained from $F_{i+1}$ by replacing the cycles in $F_{i+1}$ intersecting $D_{i+1}^{\prime}$ with the cycle

$$
u_{7} P_{1} u_{1} u_{8} P_{2} u_{4} u_{5} P_{3} u_{3} u_{2} u_{6} u_{7} .
$$

See Figure 17(d).

(iii) The ends of $P_{1}$ are $u_{7}$ and $u_{3}$.

If the ends of $P_{2}$ are $u_{8}$ and $u_{4}$, then let $F_{i}$ be the even subgraph obtained from $F_{i+1}$ by replacing the cycles in $F_{i+1}$ intersecting $D_{i+1}^{\prime}$ with the cycle

$$
u_{7} P_{1} u_{3} u_{2} u_{1} P_{3} u_{5} u_{4} P_{2} u_{8} u_{7} .
$$

See Figure 17(e).

If the ends of $P_{2}$ are $u_{8}$ and $u_{5}$, then let $F_{i}$ be the even subgraph obtained from $F_{i+1}$ by replacing the cycles in $F_{i+1}$ intersecting $D_{i+1}^{\prime}$ with the cycle

$$
u_{7} P_{1} u_{3} u_{2} u_{1} P_{3} u_{4} u_{5} P_{2} u_{8} u_{7} .
$$

See Figure 17(f).

(iv) The ends of $P_{1}$ are $u_{7}$ and $u_{4}$.

If the ends of $P_{2}$ are $u_{8}$ and $u_{5}$, then let $F_{i}$ be the even subgraph obtained from $F_{i+1}$ by replacing the cycles in $F_{i+1}$ intersecting $D_{i+1}^{\prime}$ with the cycle

$$
u_{7} P_{1} u_{4} u_{5} P_{2} u_{8} u_{1} P_{3} u_{3} u_{2} u_{6} u_{7} .
$$

See Figure $17(\mathrm{~g})$. The case that the ends of $P_{2}$ are $u_{8}$ and $u_{3}$ is the same as case (iii). See Figure 17(f).

(v) The ends of $P_{1}$ are $u_{7}$ and $u_{5}$.

If the ends of $P_{2}$ are $u_{8}$ and $u_{4}$, then let $F_{i}$ be the even subgraph obtained from $F_{i+1}$ by replacing the cycles in $F_{i+1}$ intersecting $D_{i+1}^{\prime}$ with the cycle

$$
u_{7} P_{1} u_{5} u_{4} P_{2} u_{8} u_{1} P_{3} u_{3} u_{2} u_{6} u_{7} .
$$

See Figure $17(\mathrm{~h})$. The case that the ends of $P_{2}$ are $u_{8}$ and $u_{3}$ is the same as case (iv). See Figure 17(g).

Now we completed the proof.

4. Closing remarks. The traveling salesman problem (TSP) is used to find a spanning closed walk of short length in a given graph. The typical method for TSP on 3 -edge-connected cubic graphs is as follows. First, we find a 2 -factor $F$ in a given 3edge-connected cubic graph $G$ and take a certain connected graph $T$ (e.g., a spanning tree) in the graph $G / F$ obtained from $G$ by contracting all components in $F$, and then we obtain a connected subgraph $F \cup T$ of $G$. By modifying it suitably, we can get a spanning closed walk whose length is a certain function on $|E(T)|$. Since $T$ must be a connected subgraph of $G / F,|E(T)|$ is at least the number of components of $F$ minus one, and so the lower number of components in $F$ gives the better bounds.

Aggarwal, Garg, and Gupta [1] used Theorem A to begin with a 2-factor having at most $n / 5$ components and showed the existence of a spanning closed walk of length 
at most $4 n / 3$ in a 3-edge-connected cubic graph of order $n$. This result was further improved to 2-edge connected or connected cubic graphs, graphs of maximum degree at most 3 , or better bounds than $4 n / 3$; see $[6,8,9]$.

Because of the above reasons, several researchers have been interested in a 2-factor in cubic graphs such that the number of 5-cycles is small; see [7]. Instead of using a 2-factor, we can use an even subgraph satisfying certain conditions on the order of each component. In fact, such structures have appeared in $[8,9]$ as intermediate products, which is called an $R$-factor in [8]. For those intermediate products, it is not necessarily dominating, but the dominating property may help us to obtain good bounds, i.e., we expect that Theorem 1.1 has a potential application to the TSP.

Acknowledgments. The authors would like to thank the referees for valuable suggestions and comments.

\section{REFERENCES}

[1] N. Aggarwal, N. Garg, and S. Gupta, A 4/3-Approximation for TSP on Cubic 3-EdgeConnected Graphs, arXiv:1101.5586v1, 2011.

[2] B. R. Alspach And C. D. Godsil, Eds., Cycles in Graphs, Ann. Discrete Math. 27, NorthHolland, Amsterdam, 1985.

[3] P. Ash And B. Jackson, Dominating cycles in bipartite graphs, in Progress in Graph Theory, A. Bondy and U. S. R. Murty, eds., Academic Press, New York, 1984, pp. 81-87.

[4] J. C. Bermond and C. Thomassen, Cycles in digraphs - a survey, J. Graph Theory, 5 (1981), pp. 1-43.

[5] J. A. Bondy And U. S. R. Murty, Graph Theory, Grad. Texts in Math. 244, Springer, New York, 2008.

[6] S. Boyd, R. Sitters, S. van der Ster, And L. Stougie, The traveling salesman problem on cubic and subcubic graphs, Math. Program., 144 (2014), pp. 227-245.

[7] B. Candráková and R. Lukot'Ka, Avoiding 5-circuits in 2-factors of cubic graphs, SIAM J. Discrete Math., 29 (2015), pp. 1387-1405.

[8] B. Candráková and R. LUKOT'Ka, Cubic TSP-a 1.3-Approximation, arXiv:1506.06369v1, 2015.

[9] J. Correa, O. LARré, ANd A. Soto, TSP tours in cubic graphs: Beyond 4/3, SIAM J. Discrete Math., 29 (2015), pp. 915-939.

[10] H. Fleischner, Spanning Eulerian subgraphs, the splitting lemma, and Petersen's theorem, Discrete Math., 101 (1992), pp. 33-37.

[11] H. Fleischner AND B. JaCKSOn, A note concerning some conjectures on cyclically 4-edgeconnected 3-regular graphs, in Graph Theory in Memory of G. A. Dirac, L. D. Andersen, I. T. Jakobsen, C. Thomassen, B. Toft, P. D. Vestergaard, eds., Ann. Discrete Math. 41, North-Holland, Amsterdam, 1988, pp. 171-177.

[12] D. Gamarnik, M. Lewenstein, and M. Sviridenko, An improved upper bound for the TSP in cubic 3-edge-connected graphs, Oper. Res. Lett., 33 (2005), pp. 467-474.

[13] B. JaCKSON AND K. Yoshimoto, Even subgraphs of bridgeless graphs and 2-factors of line graphs, Discrete Math., 307 (2007), pp. 2775-2785.

[14] B. JaCKSON AND K. Yoshimoto, Spanning even subgraphs of 3-edge-connected graphs, J. Graph Theory, 62 (2009), pp. 37-47.

[15] T. Kaiser And R. ŠKReKovski, Cycles intersecting edge-cuts of prescribed sizes, SIAM J. Discrete Math., 22 (2008), pp. 861-874.

[16] M. M. Matthews And D. P. Sumner, Hamiltonian results in $K_{1,3}$-free graphs, J. Graph Theory, 8 (1984), pp. 139-146.

[17] J. Petersen, Die Theorie der regulären Graphs, Acta Math., 15 (1891), pp. 193-220.

[18] Z. RYJÁČEK, On a closure concept in claw-free graphs, J. Combin. Theory. Ser. B, 70 (1997), pp. 217-224.

[19] C. Thomassen, Reflections on graph theory, J. Graph Theory, 10 (1986), pp. 309-324. 